\title{
Rendimiento de índices pronósticos en infección asociada a Clostridium difficile grave. Análisis retrospectivo en un hospital universitario
}

\author{
Cristian Hernández-Rocha, Rodrigo Tejos Sufan, Ángela Plaza-Garrido, Jonathan Barra-Carrasco, \\ Carlos Agüero Luengo, Gonzalo Inostroza Levy, Patricio Ibáñez Lazo, Ana M. Guzmán-Durán, \\ Daniel Paredes-Sabja, M. Elena Molina Pezoa y Manuel Álvarez-Lobos
}

\section{Performance of prognostic index in severe Clostridium difficile-associated infection. Retrospective analysis in a university hospital}

Introduction: By consensus severe, Clostridium difficile-associated infection (CDAI) is one that results in hospitalization in ICU, colectomy or death within 30 days. Multiple prognostic indices (IP) attempt to predict these adverse events. Objective: To evaluate the performance of 4 PI in predicting severe CDI. Methods: Hospitalized patients $\geq 18$ years old with ICD were retrospectively evaluated. Patients with recurrent infection or hematological cancer were excluded. Four PI were evaluated: UPMC version 1, Calgary version 1, Hines VA and Calgary version 2. Results: Seven of 81 patients $(8.1 \%)$ met the definition of severe CDI. Positive predicted value (PPV) and negative predicted value (NPV) of PI ranged from $20-75 \%$ and $91.3-95.7 \%$, respectively. Only Hines VA index had a satisfactory Kappa index $(0.74 ; 95 \%$ CI $0.41-1)$ with a PPV of $75 \%$ and NPV of 95,7\%. However, because of the variables included, this PI could be calculated only in $32.6 \%$ of patients. Conclusion: Hines VA index has the best predicted value and agreement to rule out a severe CDI. Like others PI it has the limitation of including difficult variables to assess in all patients and tends to overestimate an unfavorable course.

Key words: Clostridium difficile-associated infection, antibiotics-associated colitis, clinical prognostic rules, risk factors, severity, complications.

Palabras clave: Infección asociada a Clostridium difficile, colitis asociada a antimicrobianos, índices pronósticos clínicos, factores de riesgo, gravedad, complicaciones.

\section{Introducción}

$\longrightarrow$ lostridium difficile es un bacilo grampositivo, anaerobio estricto y formador de esporas, responsable de 10 a $25 \%$ de los casos de diarrea asociada a antimicrobianos y la causa identificable más común de diarrea asociada a la atención de salud ${ }^{1,2}$. Su principal mecanismo patogénico es la producción de dos exotoxinas (enterotoxina TcdA y citotoxina TcdB), responsables de un amplio espectro clínico que comprende desde una diarrea autolimitada hasta una colitis fulminante potencialmente mortal ${ }^{3}$. En las últimas dos décadas se han observado importantes cambios en la epidemiología de la infección asociada a $C$. difficile (IACD) registrándose un incremento en su incidencia, gravedad y capacidad de recurrencia ${ }^{4}$. Esto se ha asociado en parte a la aparición de cepas epidémicas como la denominada NAP1/BI/0275 recientemente identificada en Chile ${ }^{6,7}$.

En la literatura científica se encuentran múltiples definiciones de IACD grave, lo cual hace difícil la comparación de estudios diagnósticos, pronósticos y de terapia ${ }^{8,9}$.
Esto determina además, que la incidencia reportada de IACD grave sea variable, con cifras que oscilan entre 20 y $45 \%$ de los casos si se define por características clínicas como la presencia de dolor abdominal, fiebre, deterioro de la función renal o leucocitosis ${ }^{10,11}$. Sin embargo, cuando se define por la presencia de megacolon, sepsis, shock o muerte, 2 a $3 \%$ de los pacientes cursan con una IACD grave $^{12,13}$. Una reciente definición de consenso estableció que una IACD grave es aquella en la que el paciente, a causa de la infección, presenta los siguientes criterios:

- es hospitalizado en Unidad de Cuidados Intensivos (UCI) por complicaciones secundarias (ej.: shock que requiere vasopresores),

- es sometido a colectomía por perforación, megacolon o enfermedad refractaria o

- fallece dentro de los 30 días posteriores al diagnóstico ${ }^{14}$.

Esta definición tiene como objetivo estandarizar los términos usados con fines de vigilancia haciendo los reportes más comparables; no obstante, incluye eventos avanzados y ominosos en el curso de la enfermedad
Pontificia Universidad Católica de Chile, Santiago, Chile. Facultad de Medicina Departamento de Gastroenterología (CHR, RTS, CAL, PIL, MAL).

Programa de Doctorado en Ciencias Médicas (JBC).

Departamento de Cirugía

Digestiva, Unidad de

Coloproctología (GIL, MEMP).

Departamento de Laboratorio

Clínico (AMGD).

Universidad Andrés

Bello,Santiago, Chile.

Departamento de Ciencias

Biológicas (APG, DPS).

Lugar del estudio: Hospital Clínico de la Pontificia Universidad Católica de Chile.

Fuente de financiamiento: Este trabajo fue financiado por el Fondo Nacional de Ciencia y Tecnología de Chile (FONDECYT) de inicio No 11130502 de C.H.-R.; Fondo Nacional de Innovación en Salud CONICYT-FONIS SA12 |2197, FONDECYT Regular № 1131012 y del Centro de Investigaciones Médicas de la Pontificia Universidad Católica de Chile (CIM-UC PG 06/13) de M.A-L. y FONDECYT regular N 1110569, Fondos de la Vicerrectoría de Investigación de la Universidad Andrés Bello (DI-275-13/R), Fondo de Fomento al Desarrollo Científico y Tecnológico (FONDEF IdeA) CA13110077 de D.P.-S. Conflictos de interés: los autores declaran no tener conflictos de interés.

Recibido: 27 de marzo de 2014 Aceptado: 9 de octubre de 2014

Correspondencia a: Cristian Hernández-Rocha cristian.hernandez.rocha@gmail.com 
que es deseable anticipar. La detección temprana de los pacientes con riesgo de IACD grave permitiría implementar un manejo más oportuno y selectivo. En este sentido, es destacable un estudio de Zar y cols. ${ }^{11}$, que comparó la respuesta a metronidazol y vancomicina oral en IACD. Los autores observaron una respuesta similar a metronidazol o vancomicina en IACD leve, la cual fue de 90 y $98 \%$, respectivamente (p: 0,36). Sin embargo, la respuesta clínica en el grupo de pacientes con IACD grave, definida según criterios arbitrarios, fue significativamente menor para metronidazol comparado con vancomicina ( 76 vs $97 \%$, respectivamente; p: 0,02 ). Este estudio ha determinado que diferentes guías clínicas promuevan el uso de vancomicina como primera elección para cuadros de IACD grave ${ }^{15-17}$.

Factores clínicos del hospedero como edad avanzada, presencia de co-morbilidades e inmunosupresión se asocian a mayor riesgo de presentar un curso grave. Asimismo, variables como fiebre, leucocitosis, hipoalbuminemia y deterioro de la función renal se observan en cuadros de colitis por $C$. difficile más graves. Sin embargo, ninguna de estas variables por sí sola predice exactamente el curso clínico de la IACD ${ }^{18,19}$. Por esta razón, varios índices pronósticos (IP) que combinan datos clínicos, de laboratorio y radiológicos, han sido confeccionados para intentar predecir el pronóstico de la IACD, con variables rendimientos ${ }^{20}$.

El objetivo de este estudio es evaluar la capacidad de cuatro IP para predecir un curso desfavorable y determinar las variables asociadas a IACD grave en una cohorte de pacientes chilenos hospitalizados con IACD.

\section{Material y Métodos}

Se realizó una revisión de fichas clínicas de pacientes internados con IACD en el Hospital Clínico de la Pontificia Universidad Católica de Chile entre octubre del 2010 y enero del 2013. Se incluyeron pacientes hospitalizados con 18 años o más, con IACD adquirida en la comunidad o asociada a la atención de salud. Se excluyeron pacientes con episodios previos de IACD o con cáncer hematológico. Estos últimos fueron excluidos debido al efecto que tiene la enfermedad de base y la quimioterapia mielosupresora utilizada en variables como leucocitosis y creatininemia, independiente de la IACD ${ }^{21}$. Además, muchos de los estudios de derivación de los IP, excluyen a estos paciente ${ }^{20}$. Se obtuvieron antecedentes clínicos tales como: hospitalización los tres meses previos, presencia de enfermedad inflamatoria intestinal (colitis ulcerosa o enfermedad de Crohn), uso de antimicrobianos (ATM) los tres meses previos, uso de inhibidores de la bomba de protones (IBP) durante las dos semanas previas, uso actual de corticoesteroides $(5 \mathrm{mg}$ /día o más de prednisona o su equivalente) y presencia de co-morbilidades. Para evaluar la carga de co-morbilidades se calculó el índice de Charlson ${ }^{22}$ en cada paciente. El inicio de la diarrea se definió como adquirida en la comunidad o asociada a la atención de salud si ésta se iniciaba antes o después de las $48 \mathrm{~h}$ de hospitalización, respectivamente ${ }^{14}$. Se evaluó la intensidad de la diarrea con el número de deposiciones durante las $24 \mathrm{~h}$ previas al diagnóstico. Se registraron variables clínicas y de laboratorio si existían datos $24 \mathrm{~h}$ antes o $48 \mathrm{~h}$ después del diagnóstico; éstas fueron: presencia de dolor abdominal (como síntoma o signo) y su intensidad según evaluación subjetiva del médico tratante, presencia de vómitos, temperatura corporal, hipotensión arterial definida como presión arterial sistólica (PAS) menor de $100 \mathrm{mmHg}$. Se evaluaron las siguientes variables en sangre: recuento de leucocitos, proteína $\mathrm{C}$ reactiva (PCR), creatininemia, nitrógeno ureico, albuminemia, lactato y bicarbonato. Si el paciente se había realizado una tomografía axial computada (TAC) de abdomen durante el episodio de IACD se evaluó la presencia de íleo, engrosamiento de la pared del colon, megacolon y ascitis.

\section{Definiciones}

Un caso positivo de IACD fue definido como aquel que presentó tres o más episodios de diarrea las $24 \mathrm{~h}$ previas al diagnóstico más un estudio microbiológico positivo por enzimoinmunoanálisis (EIA) para toxina B y/o A(Vidas ${ }^{\circledR}$, bioMérieux, USA) o RPC para el gen $t c d B($ Cepheid $\AA$, CA, U.S.A.)(15). La utilización de estos dos métodos se debió a que durante el período de estudio se cambió la técnica diagnóstica debido a la mayor sensibilidad del examen de amplificación molecular ${ }^{23}$. Se definió una $I A C D$ grave según consenso actual, como aquella que resultó en colectomía, hospitalización en UCI o muerte dentro de 30 días, atribuibles a la IACD ${ }^{14}$. Un evaluador ciego para los resultados de los IP categorizó a los pacientes según esta definición, la cual se consideró el estándar de referencia contra el cual se compararon los IP.

Con las variables obtenidas se calcularon los siguientes IP: University of Pittsburgh Center index versión 1 (UPMC v1) $)^{24}$, University of Calgary index versión 1 (Calgary v1 $)^{25}$, Hines VA index (Hines VA) ${ }^{26}$ y University of Calgary index versión 2 (Calgary v2) ${ }^{27}$.

Las variables incluidas en cada IP y la definición de gravedad utilizada por estos se describen en la Tabla 1.

El protocolo de estudio fue aprobado por el Comité de Ética de la Pontificia Universidad Católica de Chile (Proyecto $\mathrm{N}^{\circ}$ 11-093).

\section{Análisis estadístico}

Las variables continuas fueron expresadas como media \pm desviación estándar (DE) y las variables categóricas como porcentaje. La distribución de las variables entre grupos con y sin IACD grave se evaluó a través de prueba de U Mann-Whitney para variables continuas y test exacto 


\begin{tabular}{|c|c|c|c|}
\hline Índice pronóstico & Criterios clínicos & Criterios de laboratorio y/o radiológicos & Definición de IACD grave \\
\hline UPMC V124 & Dolor abdominal (1 punto) & $\begin{array}{l}\text { Leucocitos }>20.000 / \mu \mathrm{L} o<1.500 / \mu \mathrm{L} \text { (1 punto) } \\
\text { Engrosamiento de pared intestinal, ascitis o íleo } \\
\text { en la TAC (1 punto) }\end{array}$ & $\geq 2$ puntos \\
\hline Calgary V125 & $\begin{array}{l}\text { Dolor o distensión abdominal } \geq 2 \mathrm{~h} \\
>2 \text { episodios vómitos y } T^{\circ}>38,9^{\circ} \mathrm{C} \\
>12 \text { episodios de diarrea }\end{array}$ & & $\begin{array}{l}\text { Cualquiera en las } 24 \mathrm{~h} \text { previas al diag- } \\
\text { nóstico }\end{array}$ \\
\hline Hines VA ${ }^{26}$ & $\begin{array}{l}\mathrm{T}^{\circ}>38^{\circ} \mathrm{C} \text { (1 punto) } \\
\text { Presión arterial sistólica }<100 \mathrm{~mm} \mathrm{Hg} \text { (1 punto) } \\
\text { Íleo clínico (1 punto) }\end{array}$ & $\begin{array}{l}\text { Leucocitos } \geq 15.000 / \mathrm{mm}^{3} \text { y }<30.000 / \mathrm{mm}^{3} \text { ( } 1 \text { punto) } \\
\text { Leucocitos } \geq 30.000 / \mathrm{mm}^{3} \text { ( } 2 \text { puntos) } \\
\text { Íleo radiológico ( } 1 \text { punto) } \\
\text { Hallazgos en la TAC: } \\
\text { ( } 1 \text { hallazgo }=1 \text { punto; } \geq 2 \text { hallazgos }=2 \text { puntos }) \\
\text { - Aumento de grosor pared del colon } \\
\text { - Dilatación del colon } \\
\text { - Ascitis }\end{array}$ & $\geq 3$ puntos \\
\hline Calgary V2 ${ }^{27}$ & $\begin{array}{l}\text { Dolor abdominal severo } \\
\geq 10 \text { episodios de diarrea }\end{array}$ & Leucocitosis $>20.000 / \mathrm{mm}^{3}$ & Cualquiera de las tres variables \\
\hline
\end{tabular}

de Fisher para variables categóricas. Para evaluar las variables independientemente asociadas a IACD grave se realizó análisis multivariado a través de método de regresión logística binaria, calculando el odds ratio (OR). Para todos los cálculos se consideró significativo un $p$ value $<0,05$. Para comparar el rendimientos de los IP se confeccionaron tablas de contingencia para el cálculo de sensibilidad (S), especificidad (E), valor predictor positivo (VPP) y valor predictor negativo (VPN). Se calculó el índice Kappa y su intervalo de confianza de 95\% (IC 95\%) para comparar el grado de concordancia de los 4 IP con el estándar de referencia. Un índice Kappa $>0,6$ fue considerado como un grado de concordancia satisfacto$\mathrm{rio}^{28}$. Todos los análisis fueron realizados con el programa SPSS versión 17® y Microsoft Excel ${ }^{\circledR}$.

\section{Resultados}

Ciento dieciocho casos consecutivos cumplieron con la definición de caso positivo durante el período analizado. Treinta y dos de estos episodios fueron excluidos del análisis por corresponder a cuadros recurrentes (n: 20) o ser pacientes con cáncer hematológico (n: 12). Con esto, 86 pacientes fueron incluidos en el estudio con una edad media de $62 \pm 19,1$ años; 50 pacientes $(58,1 \%)$ fueron mujeres. Siete pacientes $(8,1 \%)$ cumplieron con la definición de IACD grave, dos de estos pacientes fueron sometidos a colectomía por IACD grave refractaria y cinco requirieron hospitalización en UCI. No se registró mortalidad atribuible a IACD durante el período estudiado. Las características del total de pacientes y de los pacientes con y sin IACD grave se detallan en la Tabla 2. Sólo cuatro variables se asocia-
Tabla 2. Características demográficas, clínicas, de laboratorio y radiológicas de pacientes con IACD. Comparación entre pacientes con y sin IACD grave

\begin{tabular}{|c|c|c|c|c|}
\hline Variable & $\begin{array}{c}\text { Total } \\
(n=86)\end{array}$ & $\begin{array}{l}\text { IACD no grave } \\
(n=79)\end{array}$ & $\begin{array}{l}\text { IACD grave } \\
\quad(n=7)\end{array}$ & p-value \\
\hline Edad (años) & $62,3 \pm 19,1$ & $61,8 \pm 19,7$ & $68,1 \pm 19,7$ & NS \\
\hline Sexo femenino & $50(58,1)$ & $47(59,5)$ & $3(42,9)$ & NS \\
\hline $\begin{array}{l}\text { Antecedente de hospitalización } \\
\text { los } 3 \text { meses previos }\end{array}$ & $26(30,2)$ & $24(30,4)$ & $2(28,6)$ & NS \\
\hline Índice de Charlson & $3,5 \pm 2,6$ & $3,4 \pm 2,7$ & $5 \pm 2,4$ & NS \\
\hline Enfermedad inflamatoria intestinal & $2(2,3)$ & $1(1,3)$ & $1(14,3)$ & NS \\
\hline Uso de IBP & $31(36)$ & $28(35,4)$ & $3(42,9)$ & NS \\
\hline Uso de corticosteroides & $6(6,9)$ & $4(5,1)$ & $2(28,6)$ & NS \\
\hline Uso de ATM & $77(89,5)$ & $70(88,6)$ & $7(100)$ & NS \\
\hline Diarrea de inicio ambulatorio & $32(37,2)$ & $30(38)$ & $2(28,6)$ & NS \\
\hline Número de deposiciones en $24 \mathrm{~h}$ & $5,8 \pm 3,4$ & $5,8 \pm 3,6$ & $6 \pm 2$ & NS \\
\hline Presencia de dolor abdominal* & $30 / 59(50,8)$ & $24 / 52(46,2)$ & $6 / 7(85,7)$ & NS \\
\hline Presencia de vómitos & $8(9,3)$ & $7(8,9)$ & $1(14,3)$ & NS \\
\hline Temperatura $\geq 38^{\circ} \mathrm{C}$ & $18(20,9)$ & $11(13,9)$ & $7(100)$ & 0,0001 \\
\hline Hipotensión arterial (PAS $<100$ mmHg) & $12(14)$ & $7(8,9)$ & $5(71,4)$ & 0,0001 \\
\hline Leucocitos $\geq 15.000 / \mathrm{mm}^{3 *}$ & $21 / 72(29,1)$ & $16 / 65(24,6)$ & $5(71,4)$ & 0,02 \\
\hline Leucocitos $\geq 20.000 / \mathrm{mm}^{3 *}$ & $11 / 72(15,3)$ & $8 / 65(12,3)$ & $3(42,9)$ & NS \\
\hline Proteína $\mathrm{C}$ reactiva $(\mathrm{mg} / \mathrm{dl})$ & $10,9 \pm 11,6$ & $9,9 \pm 10,7$ & $19,8 \pm 16,4$ & NS \\
\hline Creatininemia $\geq 1,5 \mathrm{mg} / \mathrm{dl}^{*}$ & $23 / 67(34,3)$ & $19 / 61(31,1)$ & $4 / 6(66,7)$ & NS \\
\hline Creatininemia $\geq 2 \mathrm{mg} / \mathrm{dl}^{*}$ & $16 / 67(23,9)$ & $12 / 61(19,7)$ & $4 / 6(66,7)$ & 0,026 \\
\hline Nitrógeno ureico (mg/dl) & $26,4 \pm 20,7$ & $25,9 \pm 20,6$ & $31,6 \pm 22,8$ & NS \\
\hline Albuminemia $<3 \mathrm{~g} / \mathrm{dl}^{*}$ & $19 / 39(48,7)$ & $17 / 36(47,2)$ & $2 / 3(66,7)$ & NS \\
\hline Lactato (mmol/l) & $2,4 \pm 2,0$ & $1,8 \pm 1,07$ & $3,98 \pm 3,34$ & NS \\
\hline Bicarbonato (meq/l) & $19,8 \pm 4,3$ & $20,3 \pm 4,6$ & $17,5 \pm 2,1$ & NS \\
\hline TAC alterado*t & $12 / 19(63,2)$ & $8 / 15(53,3)$ & $4 / 4(100)$ & NS \\
\hline
\end{tabular}

Las variables continuas son expresadas como media \pm desviación estándar y las categóricas como $n$ (\%). *Variables categóricas calculadas con un denominador distinto debido a falta de datos en algunos


Clostridium difficile. IBP: Inhibidores de la bomba de protones. ATM: antimicrobianos. PAS: presión arterial sistólica. TAC: tomografía axial computarizada. NS: No significativo. 


\begin{tabular}{|c|c|c|c|c|c|c|}
\hline IP & $\begin{array}{c}S \\
(\%)\end{array}$ & $\begin{array}{l}E \\
(\%)\end{array}$ & $\begin{array}{l}\text { VPP } \\
\text { (\%) }\end{array}$ & $\begin{array}{l}\text { VPN } \\
\text { (\%) }\end{array}$ & $\begin{array}{l}\text { Índice Kappa } \\
\text { (IC 95\%) }\end{array}$ & $\begin{array}{l}\% \text { pacientes con } \\
\text { IP calculado }\end{array}$ \\
\hline UPMC V124 & 71,4 & 72,4 & 38,5 & 91,3 & $0,33(0,02-0,64)$ & 37,9 \\
\hline Calgary v125 & 85,7 & 47,7 & 20,7 & 95,5 & $0,14(0-0,3)$ & 53,7 \\
\hline Hines VA ${ }^{26}$ & 85,7 & 91,7 & 75,0 & 95,7 & $0,74(0,46-1)$ & 32,6 \\
\hline Calgary V227 & 85,7 & 42,9 & 20,0 & 94,7 & $0,12(0-0,27)$ & 51,6 \\
\hline
\end{tabular}

IP: Índice pronóstico. S: sensibilidad. E: especificidad. VPP: valor predictor positivo. VPN: Valor predictor negativo. consideró sólo mortalidad atribuible a IACD, ésta osciló entre 5,7 y $6,9 \%{ }^{29}$. Recientemente, con el fin de uniformar los reportes y vigilancia, se definió IACD grave como aquella en que el paciente a causa de la IACD requiere hospitalización en UCI, colectomía o fallece dentro de los 30 días posteriores al diagnóstico ${ }^{14}$. Fujitani y cols., en un estudio prospectivo de pacientes hospitalizados con IACD, utilizando estos criterios de gravedad encontró 19 casos graves en un total de 184 pacientes $(10,3 \%)^{30}$, no indicando la mortalidad registrada. Nuestro estudio, evaluando una cohorte retrospectiva y utilizando una definición similar de IACD grave, mostró una prevalencia de IACD grave discretamente menor $(8,1 \%)$. La principal variable que definió una IACD como grave en nuestro estudio fue la necesidad de hospitalización en UCI, en general secundaria a hipotensión arterial con necesidad de uso de vasopresores (cinco casos). La otra complicación registrada fue la necesidad de colectomía en dos casos donde la indicación fue megacolon tóxico que no respondió a tratamiento antimicrobiano. Ambos casos fueron refractarios al tratamiento con vancomicina oral y en enemas, además de metronidazol intravenoso y fueron sometidos a colectomía antes de cinco días del diagnóstico. No se reportó mortalidad atribuible a IACD durante los 39 meses de revisión, probablemente asociado al manejo multidisciplinario e indicación precoz de colectomía, la cual en casos graves y refractarios se ha asociado a menor mortalidad postoperatoria, sobre todo antes de alcanzar niveles de lactato sérico sobre $5 \mathrm{mmol} / \mathrm{l}^{31}$.

Estudios han demostrado que la tasa de respuesta a algunos antimicrobianos como metronidazol, utilizado habitualmente como terapia de primera línea, es menor si la IACD es grave ${ }^{11,32}$. Este hecho, junto a la mayor frecuencia de IACD grave, ha aumentado el interés en la búsqueda de factores pronósticos que permitan predecir el curso de la enfermedad. En este sentido, múltiples estudios han encontrado variables demográficas, clínicas, de laboratorio, radiológicas y endoscópicas que se asocian a mal pronóstico. Tres revisiones sistemáticas han evaluado factores de riesgo para IACD grave ${ }^{18,29,33}$; sin embargo, en ninguna fue posible realizar un meta-análisis debido a la alta heterogeneidad en el diseño de los estudios, variables consideradas, análisis estadístico de los resultados y definición de gravedad. Los factores de riesgo para IACD grave más frecuentemente reportados han sido: edad avanzada, leucocitosis, aumento de creatininemia, hipoalbuminemia y, en menor medida, el uso de corticoesteroides. Fujitani y cols., identificaron cuatro variables que se asociaron independientemente con IACD grave: distención abdominal, fiebre con $\mathrm{T}^{\circ}>38^{\circ} \mathrm{C}$, leucocitosis $\geq 20.000 / \mathrm{mm}^{3}$ y albuminemia $<3 \mathrm{~g} / \mathrm{dl}^{30}$. Al analizar nuestros datos, observamos que la mayoría de las variables previamente señaladas mostraron una tendencia a asociarse a IACD grave; no obstante, sólo la presencia de fiebre 
con $\mathrm{T}^{\circ} \geq 38^{\circ} \mathrm{C}$, PAS $<100 \mathrm{mmHg}$, leucocitosis $\geq 15.000$ $/ \mathrm{mm}^{3}$ y creatininemia $\geq 2 \mathrm{mg} / \mathrm{dl}$ se asociaron en forma estadísticamente significativa a un curso grave. El hecho que variables como hipoalbuminemia, frecuentemente señalada como factor pronóstico de IACD grave ${ }^{18,29,30,33}$ no alcanzara una asociación estadísticamente significativa podría ser explicado por el carácter retrospectivo de nuestro estudio, lo cual determina que esta variable no haya sido evaluada en todos los pacientes (sólo disponible en $58,7 \%$ de ellos), reduciendo el poder estadístico para encontrar diferencias en esta variable. En este mismo sentido, el aumento de $50 \%$ en la creatininemia, incluido en la definición de gravedad en algunas guías clínicas ${ }^{15,17}$, no pudo ser calculado debido a que pocos pacientes tenían valores basales disponibles de este parámetro (datos no mostrados). Al realizar el análisis multivariado sólo la hipotensión arterial se asoció independientemente a un desenlace grave con un OR de 23,1 (IC 95\% 1,4-372,1). Sin embargo, esta asociación podría estar determinada por la relación que existe entre esta variable y la necesidad de hospitalización en UCI, uno de los criterios que define gravedad. Esto determina que la variable PAS $<100$ $\mathrm{mmHg}$ sea menos útil para la categorización temprana de los pacientes dada su estrecha relación fisiopatológica con complicaciones mayores. No obstante, es un factor importante a tener en cuenta al evaluar pacientes con IACD ya que también estuvo presente en los dos pacientes que requirieron colectomía.

$\mathrm{Al}$ igual que en reportes previos, observamos que ninguna de las variables clínicas evaluadas precozmente es suficiente para predecir con certeza y en forma temprana el pronóstico de la IACD ${ }^{18}$. Esto ha llevado al desarrollo y evaluación de múltiples IP que permitan predecir el curso de la enfermedad. Abou Chakra y cols. ${ }^{20}$, realizaron una revisión sistemática encontrando 13 estudios de derivación de IP: dos para recurrencia, cinco para complicaciones (incluyendo mortalidad), cinco sólo para mortalidad y uno para respuesta a tratamiento, además de dos estudios de validación. Los autores encontraron múltiples limitaciones a los estudios: la mayoría fue derivada de cohortes retrospectivas y se basaron en el análisis secundario de estudios de terapia, falta de uniformidad en el momento de evaluación de las variables, variación en los outcomes reportados y falta de reporte de la incidencia de éstos. Sólo cinco de estos estudios realizaron análisis multivariado para el desarrollo del IP. Estas limitaciones ponen de manifiesto la necesidad de evaluar localmente la utilidad de los IP. Nuestro estudio es el primero en el país en realizar este análisis, evaluando el rendimiento y aplicabilidad de estas herramientas. Cabe destacar que, al igual que el meta-análisis de Abou Chakra y cols. ${ }^{20}$, la mayoría de los IP evaluados en nuestro estudio presentaron un alto VPN con valores entre 91,3 y 95,7\% lo cual los hace herramientas útiles para descartar un curso grave. Sin embargo, el VPP de los IP evaluados es sub-óptimo (20 a 75\%), lo cual redunda en una excesiva predicción de cuadros graves que no presentarán un curso desfavorable con el consecuente aumento innecesario de recursos terapéuticos y costos. El índice de Hines VA que incluye tres de las variables que en nuestro estudio alcanzaron una asociación estadísticamente significativa (hipotensión arterial, fiebre y leucocitosis), presentó el más alto VPP, aunque sigue siendo sub-óptimo (75\%). Estos datos de rendimiento de los IP son concordantes con estudios previos ${ }^{20,30}$. Un dato destacable de nuestro estudio no señalado en reportes previos, es que, en un número importante de pacientes, los IP no pueden ser calculados sobre todo cuando incluyen variables radiológicas. En sólo 19 pacientes (22\%) se realizó una TAC de abdomen, principalmente en casos complicados o con diagnóstico dudoso. Así, el índice de Hines VA, que presentó el mejor rendimiento, sólo pudo ser calculado en $32,6 \%$ de los pacientes. Este hecho además determinó que otros IP publicados ${ }^{30}$ no pudieran ser calculados, ya que un escaso número de pacientes tenía medidas las variables necesarias para su estimación (datos no mostrados). Con el advenimiento de nuevos métodos diagnósticos de alto rendimiento y no invasores, la utilización de exámenes radiológicos o endoscópicos para casos de diagnóstico dudoso se hace menos probable, con lo cual la utilidad clínica de éstos será reservada para pacientes que cursan con una IACD ya complicada. Esto hace poco práctica su utilización en IP de aplicación temprana en el curso de la enfermedad, por lo que nuevas herramientas de predicción no deberían incluir estas variables. Nuevos IP deberían construirse a partir de variables evaluables tempranamente en múltiples grupos de pacientes independiente de sus co-morbilidades.

La exclusión de grupos con alto riesgo de IACD como aquellos con neoplasia hematológica, donde la enfermedad o sus tratamientos alteran variables importantes como leucocitosis y creatininemia ${ }^{21}$, disminuye la aplicabilidad de los IP actuales. En este sentido, el estudio de nuevas variables genéticas y/o adquiridas del hospedero asociadas a la respuesta inflamatoria o el estudio de factores patogénicos de $C$. difficile asociados a IACD grave se vuelven de alto valor ${ }^{34,35}$.

En resumen, actualmente no existen variables individuales que puedan predecir en forma temprana y certera una IACD grave. Los IP diseñados a la fecha, si bien muestras un buen VPN, carecen de una adecuada especificidad; sin embargo, su principal limitación está en la utilización de variables que son difíciles de evaluar en todos los pacientes con IACD lo que reduce su aplicabilidad en la práctica clínica. Ante el nuevo escenario epidemiológico, es fundamental desarrollar nuevas herramientas que permitan evaluar precozmente el pronóstico de los pacientes con IACD. Éstas deberían incluir variables 
clínicas o de laboratorio objetivas, no invasoras y fáciles de medir. Por último, es fundamental que estas variables sean evaluadas en estudios prospectivos bien diseñados, que incluyan la predicción de eventos clínicos relevantes y definiciones consensuadas de IACD grave.

\section{Resumen}

Introducción: Por consenso, la infección asociada a Clostridium difficile (IACD) grave es aquella que resulta en hospitalización en unidad de cuidados intensivos, colectomía o muerte dentro de 30 días. Múltiples índices pronósticos (IP) intentan predecir estos eventos adversos. Objetivo: evaluar el rendimiento de cuatro IP en la predicción de IACD grave. Metodología: pacientes hospitalizados $\geq 18$ años con IACD fueron evaluados retrospectivamente. Se excluyeron pacientes con infección recurrente o cáncer hematológico. Se evaluaron cuatro IP: UPMC versión 1, Calgary versión 1, Hines VA y Calgary versión 2. Resultados: Siete de 81 pacientes $(8,1 \%)$ presentaron una IACD grave. El valor predictor positivo (VPP) y valor predictor negativo (VPN) de los IP varió entre $20-75 \%$ y 91,3-95,7\%, respectivamente. Sólo el índice de Hines VA tuvo un índice Kappa satisfactorio (0,74; IC 95\% 0,46-1) con un VPP de $75 \%$ y un VPN de $95,7 \%$. Sin embargo, por las variables incluidas en este IP, sólo pudo ser calculado en $32,6 \%$ de los pacientes. Conclusión: El índice de Hines VA presenta el mejor valor predictor y concordancia para descartar una IACD grave. Como otros IP, tiene la limitación de incluir variables difícilmente evaluables en todos los pacientes y tiende a sobreestimar un curso desfavorable.

\section{Referencias bibliográficas}

1.- Bartlett J G. Clostridium difficile: history of its role as an enteric pathogen and the current state of knowledge about the organism. Clin Infect Dis 1994;18 Suppl 4: S265-72.

2.- Barbut F, Corthier G, Charpak Y, Cerf M, Monteil H, Fosse T, et al. Prevalence and pathogenicity of Clostridium difficile in hospitalized patients. A French multicenter study. Arch Intern Med 1996; 156 (13): 1449-54.

3.- Bartlett J G. Clinical practice. Antibioticassociated diarrhea. N Engl J Med 2002; 346 (5): 334-9.

4.- Freeman J, Bauer M P, Baines S D, Corver J, Fawley W N, Goorhuis B, et al. The changing epidemiology of Clostridium difficile infections. Clin Microbiol Rev 2010; 23 (3): 529-49.

5.- Clements A C, Magalhaes R J, Tatem A J, Paterson D L, Riley T V. Clostridium difficile PCR ribotype 027: assessing the risks of further worldwide spread. Lancet Infect Dis 2010; 10 (6): 395-404.

6.- Hernández-Rocha C, Barra-Carrasco J, Pizarro-Guajardo M, Ibáñez P, Bueno S M, Sarker M R, et al. Epidemic Clostridium difficile ribotype 027 in Chile. Emerg Infect Dis 2012; 18 (8): 1370-2.

7.- Barra-Carrasco J, Hernández-Rocha C, Miranda-Cárdenas C, Álvarez-Lobos M, Guzmán Durán A M, Paredes-Sabja D. Diagnostic accuracy of a multiplex real-time PCR to predict Clostridium difficile ribotype 027. Anaerobe 2013; 22: 115-7.

8.- Surawicz C M, Alexander J. Treatment of refractory and recurrent Clostridium difficile infection. Nat Rev Gastroenterol Hepatol 2011; 8 (6): 330-9.

9.- Hernandez-Rocha C. Clasificación de gravedad en infección por Clostridium difficile. Gastroenterol Latinoam 2013; 24 (4): 214-7.

10.- Khanna S, Pardi D S, Aronson S L, Kammer P P, Orenstein R, St Sauver J L, et al. The epidemiology of community-acquired Clostridium difficile infection: a populationbased study. Am J Gastroenterol 2012; 107 (1): 89-95.

11.- Zar F A, Bakkanagari S R, Moorthi K M, Davis M B. A comparison of vancomycin and metronidazole for the treatment of Clostridium difficile-associated diarrhea, stratified by disease severity. Clin Infect Dis 2007; 45 (3): 302-7.

12.- Miller MA, Hyland M, Ofner-Agostini M, Gourdeau M, Ishak M. Morbidity, mortality, and healthcare burden of nosocomial Clostridium difficile-associated diarrhea in Canadian hospitals. Infect Control Hosp Epidemiol 2002; 23 (3): 137-40.

13.- Rubin M S, Bodenstein L E, Kent K C. Severe Clostridium difficile colitis. Dis Colon Rectum 1995; 38 (4): 350-4.

14.- McDonald LC, Coignard B, Dubberke E, Song X, Horan T, Kutty P K. Recommendations for surveillance of Clostridium difficileassociated disease. Infect Control Hosp Epidemiol 2007; 28 (2): 140-5.

15.- Cohen S H, Gerding D N, Johnson S, Kelly C P, Loo V G, McDonald L C, et al. Clinical practice guidelines for Clostridium difficile infection in adults: 2010 update by the Society for Healthcare Epidemiology of America (SHEA) and the Infectious Diseases Society of America (IDSA). Infect Control Hosp Epidemiol 2010; 31 (5): 431-55

16.- Surawicz C M, Brandt L J, Binion D G, Ananthakrishnan A N, Curry S R, Gilligan P H, et al. Guidelines for diagnosis, treatment, and prevention of Clostridium difficile infections.
Am J Gastroenterol 2013; 108 (4): 478-98; quiz 99.

17.- Debast SB, Bauer MP, Kuijper EJ. European Society of Clinical Microbiology and Infectious Diseases: update of the treatment guidance document for Clostridium difficile infection. Clin Microbiol Infect. 2014; 20 Suppl 2: $1-26$.

18.- Bloomfield MG, Sherwin JC, Gkrania-Klotsas E. Risk factors for mortality in Clostridium difficile infection in the general hospital population: a systematic review. J Hosp Infect. 2012; 82(1): 1-12.

19.- Henrich TJ, Krakower D, Bitton A, Yokoe DS. Clinical risk factors for severe Clostridium difficile-associated disease. Emerg Infect Dis. 2009; 15(3): 415-22.

20.- Abou Chakra CN, Pepin J, Valiquette L. Prediction tools for unfavourable outcomes in Clostridium difficile infection: a systematic review. PLoS One. 2012;7(1): e30258.

21.- Wang MS, Evans CT, Rodriguez T, Gerding DN, Johnson S. Clostridium difficile infection and limitations of markers for severity in patients with hematologic malignancy. Infect Control Hosp Epidemiol. 2013; 34(2): 127-32.

22.- Charlson ME, Pompei P, Ales KL, MacKenzie CR. A new method of classifying prognostic comorbidity in longitudinal studies: development and validation. J Chronic Dis. 1987; 40(5): 373-83.

23.- Hernandez-Rocha C, Barra-Carrasco J, AlvarezLobos M, Paredes-Sabja D, Guzman-Duran AM. Prospective comparison of a commercial multiplex real-time polymerase chain reaction and an enzyme immunoassay with toxigenic culture in the diagnosis of Clostridium difficileassociated infections. Diagn Microbiol Infect Dis. 2013; 75(4): 361-5.

24.- McEllistrem MC, Carman RJ, Gerding DN, 
Genheimer CW, Zheng L. A hospital outbreak of Clostridium difficile disease associated with isolates carrying binary toxin genes. Clin Infect Dis 2005; 40 (2): 265-72.

25.- Louie TJ, Peppe J, Watt CK, Johnson D, Mohammed R, Dow G, et al.Tolevamer, a novel nonantibiotic polymer, compared with vancomycin in the treatment of mild to moderately severe Clostridium difficileassociated diarrhea. Clin Infect Dis 2006; 43 (4): 411-20.

26.- Belmares J, Gerding D N, Parada J P, Miskevics S, Weaver F, Johnson S. Outcome of metronidazole therapy for Clostridium difficile disease and correlation with a scoring system. J Infect 2007; 55 (6): 495-501.

27.- Louie. Results of a phase III trial comparing tolevamer, vancomycin and metronidazole in Clostridium difficile-associated diarrhea (CDAD). 47th Interscience Conference on
Antimicrobial Agents and Chemotherapy. Washington DC: American Society for Microbiology; 2007: Abstract K-425a.

28.- Sim J, Wright CC. The kappa statistic in reliability studies: use, interpretation, and sample size requirements. Phys Ther 2005; 85 (3): 257-68

29.- Mitchell B G, Gardner A. Mortality and Clostridium difficile infection: a review. Antimicrob Resist Infect Control 2012; 1 (1): 20.

30.- Fujitani S, George W L, Murthy A R. Comparison of clinical severity score indices for Clostridium difficile infection. Infect Control Hosp Epidemiol 2011; 32 (3): 220-8.

31.- Lamontagne F, Labbe A C, Haeck O, Lesur O, Lalancette M, Patino C, et al. Impact of emergency colectomy on survival of patients with fulminant Clostridium difficile colitis during an epidemic caused by a hypervirulent strain. Ann Surg 2007; 245 (2): 267-72.

32.- Musher D M, Aslam S, Logan N, Nallacheru S, Bhaila I, Borchert F, et al. Relatively poor outcome after treatment of Clostridium difficile colitis with metronidazole. Clin Infect Dis 2005; 40 (11): 1586-90.

33.- Karas J A, Enoch D A, Aliyu S H. A review of mortality due to Clostridium difficile infection. J Infect 2010; 61 (1): 1-8.

34.- Viladomiu M, Hontecillas R, Pedragosa M, Carbo A, Hoops S, Michalak P, et al. Modeling the role of peroxisome proliferator-activated receptor gamma and microRNA-146 in mucosal immune responses to Clostridium difficile. PLoS One 2012; 7 (10): e47525.

35.- Walker A S, Eyre D W, Wyllie D H, Dingle K E, Griffiths D, Shine B, et al. Relationship between bacterial strain type, host biomarkers, and mortality in Clostridium difficile infection. Clin Infect Dis 2013; 56 (11): 1589-600. 